\title{
Improving Tensile Properties of Polylactic Acid Parts by Adjusting Printing Parameters of Open Source 3D Printers
}

\author{
Yubo TAO, Peng LI*, Ling PAN \\ College of Material Science and Engineering, Northeast Forestry University, Harbin, Heilongjiang 150040, China \\ crossref http://dx.doi.org/10.5755/j01.ms.26.1.20952
}

Received 15 June 2018; accepted 12 October 2018

\begin{abstract}
Open source 3D printers (OS3DPs) have become increasingly more widespread in recent years because of their ease of use and budget friendliness, with the majority being fused deposition modeling (FDM) printers. However, due to natural deficiencies of the FDM printing methodology, different printing parameters can cause various properties of printed parts. To obtain printed polylactic acid (PLA) parts with improved tensile properties, a tension model of the part and an orthogonal experiment scheme were constructed in this paper. The effects of three printing parameters (layer height, orientation angle (OA) of the part, and print speed) on tensile properties (elastic modulus, tensile strength, and elongation at break) were investigated. The results demonstrated that the printing parameters affected the tensile properties of PLA parts. Larger layer height and lower print speed contributed to the improvement of tensile strength. The OA of the part had the greatest effect on the parts' elastic modulus and elongation at break among the three parameters. Both layer height and OA of the part affected its tensile strength significantly. In this research, the layer height of $0.2 \mathrm{~mm}$ and the print speed of $20-30 \mathrm{~mm} / \mathrm{s}$ were found to be the optimal printing parameters. Adjusting the $\mathrm{OA}$ of the part can provide targeted tensile properties, and the parts with the OA of $45^{\circ}$ resulted in the lowest tensile strength because the tensile force was only held by fibers parallel to the force orientation instead of all fibers. Keywords: fused deposition modelling, 3D printing, tensile properties, open source 3D printers.
\end{abstract}

\section{INTRODUCTION}

Additive manufacturing (AM) is defined as "a process of joining materials to make objects from threedimensional (3D) model data, usually layer upon layer, as opposed to subtractive manufacturing methodologies" [1]. Expiration of early additive manufacturing patents leads to the growth of relatively low-cost, open source 3D printers (OS3DPs), such as Ultimate and RepRaps (selfREPlicating and RAPid) in the past decade [2, 3]. OS3DPs have given hobbyists and enthusiasts the chance to adopt the do-it-yourself (DIY) culture and experiment with their own designs without computerized numerical control machining knowledge.

OS3DPs usually adopt a manufacturing method known as fused deposition modeling (FDM) to build physical objects from 3D digital models using thermoplastic filaments such as acrylonitrile butadiene styrene (ABS) or polylactic acid (PLA). ABS is a common thermoplastic polymer, manufactured by polymerizing styrene and acrylonitrile in the presence of polybutadiene. PLA, a compostable synthetic polymer produced using monomer feedstock derived from corn starch, satisfies many of the environmental impact criteria required for an acceptable replacement for oil-derived plastics [4].

A digital model (typically STL format file) is required for $3 \mathrm{D}$ printing. Created using CAD software or scanned by $3 \mathrm{D}$ scanners, the model is sliced into horizontal layers with each layer representing a cross-sectional contour of the model based on calculations by 3D printing software. $3 \mathrm{D}$ printing software, such as the CURA (developed by

\footnotetext{
${ }^{*}$ Corresponding author. Tel.: +86-451-82190394;

E-mail address: lptyb@aliyun.com (P. Li)
}

Ultimaker), also enables the customization of printing parameters for users to calibrate their software (and printers). As shown in Fig. 1, in the FDM process, thermoplastic filament is fed to the heating element with the pressure generated from the filament feeding component. The filament material is heated to melting state and the fiber is extruded through the extrusion nozzle. The extrusion nozzle deposits the material (fiber) on the $\mathrm{X}-\mathrm{Y}$ plane following the software generated tool paths. Fibers are bonded to form a whole. Either the print bed or the extrusion nozzle will move to provide necessary space for the next layer's fabrication. Every single layer will be stacked onto the previous until the part is completed [5].

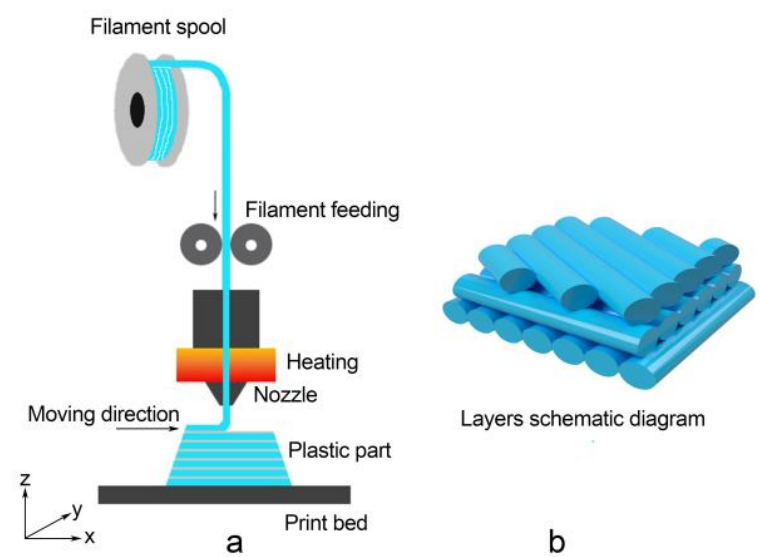

Fig. 1. The FDM process relies on a heated nozzle that spatially distributes extruded polymer in a layer-by-layer approach: $a-F D M$ printing mechanics; $b$-layers schematic diagram 
Due to the fact that most OSDPs deposit material in a directional way (as illustrated in magnified layer schematic diagram of Fig. $1 \mathrm{~b}$ ), the FDM process produces plastic parts with anisotropic behaviours [6-8]. Previous studies have been conducted to observe the effects of parameters on the mechanical properties of FDM printed products. Ahn et al. investigated the effects of air gap, raster width, nozzle temperature, ABS colour, and raster angle on the tensile strength. They found that air gap and raster angle significantly affected the tensile strength. When the raster angle was $0^{\circ}$ (parallel to loading direction), the tensile strength was the highest; on the contrary, when the raster angle was $90^{\circ}$ (perpendicular to the loading direction), the tensile strength was the lowest. Though decreasing the air gap to a negative value can increase the tensile strength, but the material overlap can result in surface roughness [9]. Bellini et al. concluded that the mechanical properties of the parts depended on the orientation of the object with respect to the substrate and the way roads filled every layer [10]. Sood et al. developed the empirical models relating responses (tensile, flexural, and impact strength) and five process parameters: layer thickness, orientation, raster angle, raster width, and air gap. Effects of various parameters and their interactions were analyzed using response surface plots and the optimal parameter setting for each response was determined [11]. Based on an FDM desktop printer, Lanzotti et al. studied the effects of layer thickness, raster angle, and shell perimeters on the strength of $3 \mathrm{D}$ printed parts and maximized the ultimate tensile strength by optimizing the parameters [12]. Dawoud et al. investigated the effects of printing parameters on the mechanical behaviours of ABS part samples that were made by an injection molding process or FDM process. Their research revealed that FDM parts produced with a negative air gap gained higher density and enhanced mechanical behaviour than those with a positive air gap [13].

According to the literature, air gap and raster orientation show more significant effects on the mechanical performance than layer thickness, bead width, colour, etc. However, the adjusting of air gap and raster orientation is not well suited for average users. Thus, parameters from the basic settings were investigated in this study. Common users often struggle to find a balance between sacrificing print efficiency, which is affected by layer height and print speed, for mechanical properties and vice versa. Although parameters such as raster angle and air gap cannot be adjusted through basic settings, users can easily modify the layer height and the print speed to optimize the print efficiency. Whereas for configuring a part's mechanical properties, this paper focuses on the part orientation angle (OA) for the sake of user convenience.

In the present work, we analyzed the tension model of the PLA parts and explored effects of printing parameters (layer height, print speed, and OA of the part on the internal structure and mechanical properties of printed parts. Furthermore, we optimized the printing parameters in CURA for part tensile properties (elastic modulus, tensile strength, and elongation at break) using orthogonal experiment design.

\section{EXPERIMENTAL}

\subsection{Tension model of the plastic part}

Due to the nature of the FDM printing process, a printed part's tensile properties vary with the extruded fiber's cross-section contour and area. As shown in Figure $2 \mathrm{a}$, assuming that the extruded fiber is in the shape of an ellipse, thereupon, the relation below applies:

$\mathrm{A}_{e l}=\frac{1}{4} \pi w h$

$\mathrm{A}_{c i}=\frac{1}{4} \pi d^{2}$;

$Q=\mathrm{A}_{e l} v_{p}=\frac{1}{4} \pi w h v_{p} ;$

$Q=\mathrm{A}_{c i} v_{e}=\frac{1}{4} \pi d^{2} v_{e}$

$\emptyset=\frac{\mathrm{nA}_{e l}}{A_{c p}}$,

where $\mathrm{A}_{e l}$ is the area of fiber cross-section ellipse, $\mathrm{mm}^{2}$; $\mathrm{A}_{c i}$ is the area of nozzle, $\mathrm{mm}^{2} ; \mathrm{A}_{c p}$ is the cross-section area of part, $\mathrm{mm}^{2} ; Q$ is the volume of filament used per second, $\mathrm{mm}^{3} / \mathrm{s} ; w$ is the long axis length of fiber cross-section ellipse, $\mathrm{mm}$; $h$ is the short axis length of fiber cross-section ellipse, $\mathrm{mm}$; $d$ is the diameter of nozzle, $\mathrm{mm} ; v_{e}$ is the velocity of extrusion fiber, $\mathrm{mm} / \mathrm{s} ; v_{p}$ is the velocity of print speed, $\mathrm{mm} / \mathrm{s} ; \phi$ is the cross-section porosity of part.

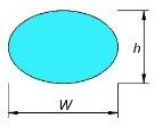

a
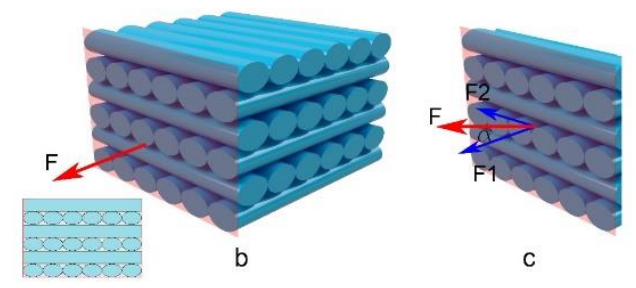

C
Fig. 2. Tension forces of plastic part: a-cross-section of a single strand fiber in the part; $b$-diagram of the tension forces on the cross-section of part, the tension force is parallel or perpendicular to the fibers; $\mathrm{c}-$ an angle of $\alpha$ exists between the tension force and fiber orientation

Fig. 2 b illustrates the tension force on cross-section of the part. The tension force is parallel to the fiber (or perpendicular to the fiber in next layer) and is sustained primarily by fibers parallel to its direction. Fig. $2 \mathrm{c}$ demonstrates that another directional relationship between tension force and fiber, an angle of $\alpha$ exists between the tension force direction and fiber orientation.

\subsection{Preparation of PLA parts}

The PLA parts were designed according to ASTM D638 [14]. The specific size of the test specimen is described in Fig. 3. PLA filaments of $1.75 \mathrm{~mm}$ diameter were printed as test specimens using an OS3DP (Printing feedstock and 605S model machine from Shenzhen Aurora Technology Co, Shenzhen, China). The nozzle diameter of printer was $0.4 \mathrm{~mm}$. The CURA software (15.04 version, developed by Ultimaker) was used to set printing parameters. The printing temperature was $210{ }^{\circ} \mathrm{C}$ and the fill density was $100 \%$. 


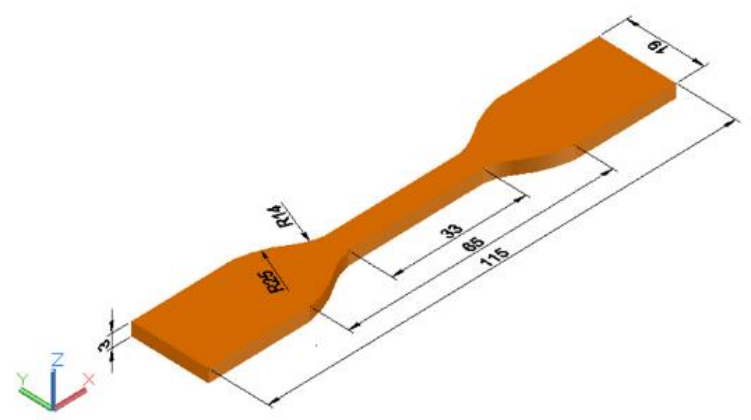

Fig. 3. Specific size and shape of printed parts

\subsection{Tensile properties measurement of parts}

Tensile properties of the specimens were measured by a universal testing machine (Changchun Kexin instruments Co. Changchun, China). The testing speed was set to $25 \mathrm{~mm} / \mathrm{min}$. Elasticity modulus, tensile strength, and elongation at break were obtained according to ASTM D638.

\subsection{Orthogonal experiment design}

Three printing parameters of the CURA software, including layer height, OA of the part, and print speed, were selected as fixed factors. Each fixed factor has three levels and every level repeats three times to have a mean value. In the CURA software, layer height is the thickness of each deposited layer; print speed is the moving speed of nozzle along the $\mathrm{X}$ and $\mathrm{Y}$-axis; and the $\mathrm{OA}$ of the part represents the angle between the central axis of the PLA part and the $\mathrm{X}$ coordinate in the software, as shown in Fig. 4. The level values of fixed factors are listed in Table 1. Orthogonal experiment scheme of $\mathrm{L}_{9}\left(3^{4}\right)$ was designed according to the number of factors and levels. The dependent variables are elastic modulus, tensile strength, and elongation at break. The experimental scheme is shown in Table 2.

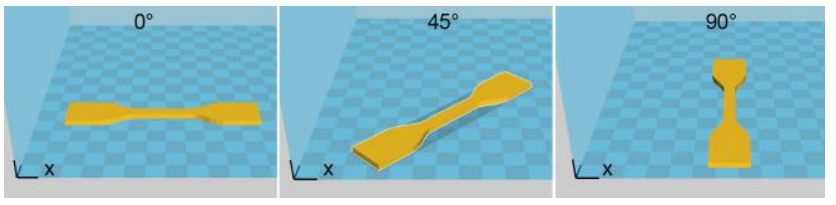

Fig. 4. Orientation angles (OA) of PLA parts

Table 1. Factors and levels of experiments

\begin{tabular}{|c|c|c|c|}
\hline \multirow{2}{*}{ Levels } & \multicolumn{3}{|c|}{ Fixed factors } \\
\cline { 2 - 4 } & $\begin{array}{c}\text { Layer height, } \\
\mathrm{mm}\end{array}$ & $\begin{array}{c}\text { OA of the part, } \\
\circ\end{array}$ & $\begin{array}{c}\text { Print speed, } \\
\mathrm{mm} / \mathrm{s}\end{array}$ \\
\hline 1 & 0.1 & 0 & 20 \\
\hline 2 & 0.2 & 45 & 30 \\
\hline 3 & 0.3 & 90 & 40 \\
\hline
\end{tabular}

\section{RESULTS AND DISCUSSION}

\subsection{Calculated porosity and force analysis of cross-section area in part}

As shown in Fig. 5, under the same conditions, with the increase of layer height, the calculated porosity decreased, whereas, with the increase of print speed, calculated porosity increased. For parts with the same size, smaller porosity of the cross-section resulted in higher density and greater force that the part could sustain. This is consistent with the previous researches that decreased air gap can increase the density of parts and improve their strength. Therefore, increasing layer height and decreasing print speed will be beneficial in improving tensile properties theoretically.

Table 2. Orthogonal experiment scheme

\begin{tabular}{|c|c|c|c|c|c|}
\hline \multirow{2}{*}{ Test } & A & B & C & D & \multirow{2}{*}{ Conditions } \\
\cline { 2 - 5 } & $\begin{array}{c}\text { Layer } \\
\text { height }\end{array}$ & $\begin{array}{c}\text { OA of } \\
\text { the part }\end{array}$ & Null & $\begin{array}{c}\text { Print } \\
\text { speed }\end{array}$ & \\
\hline 1 & $1(0.1)$ & $1(0)$ & 1 & $1(20)$ & A1B1C1D1 \\
\hline 2 & $1(0.1)$ & $2(45)$ & 2 & $2(30)$ & A1B2C2D2 \\
\hline 3 & $1(0.1)$ & $3(90)$ & 3 & $3(40)$ & A1B3C3D3 \\
\hline 4 & $2(0.2)$ & $1(0)$ & 2 & $3(40)$ & A2B1C2D3 \\
\hline 5 & $2(0.2)$ & $2(45)$ & 3 & $1(20)$ & A2B2C3D1 \\
\hline 6 & $2(0.2)$ & $3(90)$ & 1 & $2(30)$ & A2B3C1D2 \\
\hline 7 & $3(0.3)$ & $1(0)$ & 3 & $2(30)$ & A3B1C3D2 \\
\hline 8 & $3(0.3)$ & $2(45)$ & 1 & $3(40)$ & A3B2C1D3 \\
\hline 9 & $3(0.3)$ & $3(90)$ & 2 & $1(20)$ & A3B3C2D1 \\
\hline
\end{tabular}

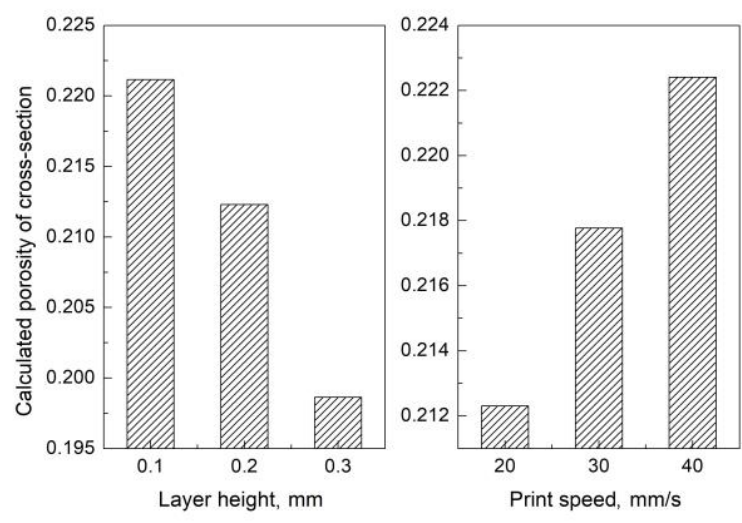

Fig. 5. Layer height and print speed's effects on cross-section porosity of parts

When the tension force $\mathrm{F}$ is parallel or perpendicular to the fibers, illustrated by Fig. 2 b, most of tension force would be sustained by fibers that are parallel to force F, especially when air gaps exist between those perpendicular fibers. While the tension force $F$ is at an angle to the fibers, illustrated by Fig. 2 c, the relation $F 1=F \cdot \cos (\alpha)$ applies. Due to the fact that $F 1$ is a component force, consequently, the actual tension force along the fiber orientation is smaller than $F$, with a corresponding rise of the tensile strength.

\subsection{Relationship among OA, tension force direction and fiber orientation}

Through nozzle movement, FDM deposits fibers (molten filament of PLA) in a crisscross manner. Fibers are bonded to form a whole. Therefore, the direction of the fibers will affect tensile properties of the part. Because users cannot control fiber orientation, $\mathrm{OA}$ is used to get various fiber orientations. Fig. 6 shows the differences between the fiber arrangements at different direction angles inside the parts. As illustrated by Fig. 6, in the CURA software, the nozzle's infill movement is limited to $45^{\circ}$ and $135^{\circ}$ to the $\mathrm{x}$-axis. Thus, the same fiber orientation 
could be observed within $\mathrm{OA} 90^{\circ}$ and $0^{\circ}$ because of the fixed nozzle moving path. When the OA of the part is $45^{\circ}$, the direction of tensile force is parallel to the fiber orientation (perpendicular to the fiber orientation in the next layer), shown in Fig. 2 b. However, when OA is either $0^{\circ}$ or $90^{\circ}$, an angle of $\alpha\left(\alpha=45^{\circ}\right)$ exists between the tension force and the fiber orientation, as shown in Fig. 2 b.
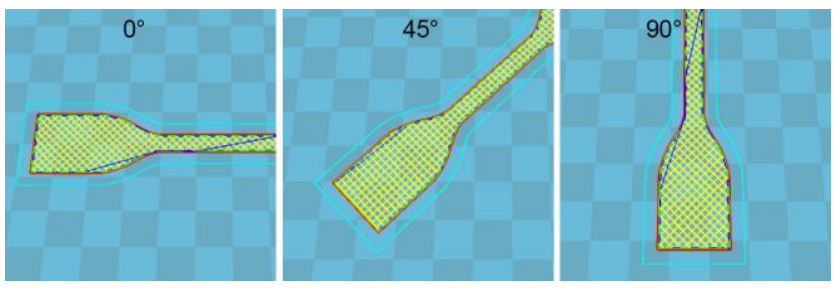

Fig. 6. The moving path of the nozzle under different OAs (yellow line represents moving path and fiber cross direction)

\subsection{Orthogonal experiment}

The results of each test were analyzed using SPSS (IBM SPSS Statistics 19). Table 3 represents the contributions of the level change of fixed factors to the dependent variables response. According to the orthogonal experiment method, the greater the $F$ value of fixed factors, the greater impacts on the dependent variables response.

Elastic modulus (MOE): Comparing layer height, OA of part, and print speed, the effect of the OA on the elastic modulus was the greatest $(F=2.477)$, followed by the layer height and the print speed. Elastic modulus represents a specimen's elastic deformity resistance upon an applied force. A stiffer material has a higher elastic modulus. Thus, the OA of the part has a substantial effect on part stiffness.

Table 3. Tests of between-subject effects (calculated by SPSS)

\begin{tabular}{|c|c|c|c|c|}
\hline $\begin{array}{c}\text { Dependent } \\
\text { variables }\end{array}$ & Fixed factors & $\begin{array}{c}\text { Mean } \\
\text { square }\end{array}$ & F & Sig. \\
\hline \multirow{3}{*}{ MOE } & Layer height & 25381.951 & 1.838 & 0.352 \\
\cline { 2 - 5 } & OA of the part & 34209.521 & $\mathbf{2 . 4 7 7}$ & 0.288 \\
\cline { 2 - 5 } & Print speed & 15486.101 & 1.122 & 0.471 \\
\hline \multirow{3}{*}{ TS } & Layer height & 62.715 & $\mathbf{3 6 . 3 4 8}$ & $\mathbf{0 . 0 2 7}$ \\
\cline { 2 - 5 } & OA of the part & 35.619 & 20.644 & $\mathbf{0 . 0 4 6}$ \\
\cline { 2 - 5 } & Print speed & 13.924 & 8.070 & 0.110 \\
\hline \multirow{3}{*}{ EL } & Layer height & 0.412 & 0.402 & 0.713 \\
\cline { 2 - 5 } & OA of the part & 7.700 & $\mathbf{7 . 5 1 7}$ & 0.117 \\
\cline { 2 - 5 } & Print speed & 0.449 & 0.438 & 0.695 \\
\hline
\end{tabular}

Tensile strength (TS): measured by the maximum stress that a specimen can withstand while being stretched or pulled before breaking. The analysis showed that the layer height had the greatest effect $(F=36.348)$ on tensile strength compared with the OA of the part and the print speed. Higher layer height means bigger diameter for each single fiber, lower cross-section porosity, and better tensile strength. Notably, the Sig. values of layer height (0.027) and OA (0.046) were all below 0.05 , which indicates that they have a significant impact on the tensile strength of PLA parts.

Elongation at break (EL): also known as fracture strain, is the ratio between stretched length (difference between final length and initial length) and initial length after breakage of the test specimen. The value of elongation implies the capacity of a specimen to resist changes in shape without cracking its formation. The influence of fixed factors on the elongation from high to low is OA of the part, print speed, and layer height. It can be seen that among three parameters, the OA of the part is the most important factor for forming the area of stress concentration in the PLA parts.

\subsection{Optimized printing parameters}

Fig. 7 illustrates the Duncan multiple comparison results between levels of fixed factors.

According to the results, optimal printing parameters for the highest elastic modulus, tensile strength, and elongation, are listed in Table 4.

Table 4. Summary of optimized printing parameters

\begin{tabular}{|l|c|c|c|}
\hline Targets & $\begin{array}{c}\text { Layer height, } \\
\mathrm{mm}\end{array}$ & $\begin{array}{c}\text { OA of the } \\
\text { part, }\end{array}$ & $\begin{array}{c}\text { Print speed, } \\
\mathrm{mm} / \mathrm{s}\end{array}$ \\
\hline MOE & 0.3 & 45 & 20 \\
\hline TS & 0.3 & 0 & 30 \\
\hline EL & 0.3 & 90 & 20 \\
\hline
\end{tabular}

Larger layer height was proven to be helpful for enhancing tensile properties, however, harmful to surface quality. The parts with the $\mathrm{OA}$ of $45^{\circ}$ resulted in the lowest tensile strength because the tensile loads were only held by fibers parallel to the loading direction instead of all fibers. The print speed of $40 \mathrm{~mm} / \mathrm{s}$ is not recommended although it can improve print efficiency.

\section{CONCLUSIONS}

From the model analysis and orthogonal experiment design of FDM PLA using an OS3DP (The CURA software was used to set printing parameters), conclusions can be drawn:

1. PLA Parts with specific tensile properties can be obtained by using different printing parameters.

2. OA of the part has greater effect on elastic modulus and elongation at break than layer height and print speed because of various fiber orientations.

3. Layer height and OA of the part greatly affect the tensile strength of a PLA part. When tensile loads were only held by fibers parallel to the loading direction instead of all fibers can result in the lowest tensile strength.

4. $0.2 \mathrm{~mm}$ of layer height and $20-30 \mathrm{~mm} / \mathrm{s}$ of print speed are optimal printing parameters based on our research.

\section{Acknowledgments}

Supported by the Fundamental Research Funds for the Central Universities (2572019bb07). The authors would like to thank Zelong $\mathrm{Li}$ for his technical support and designing figures.

\section{REFERENCES}

1. ASTM F2792-12a. Standard Terminology for Additive Manufacturing Technologies. ASTM International, West Conshohocken, PA, USA, 2012. 

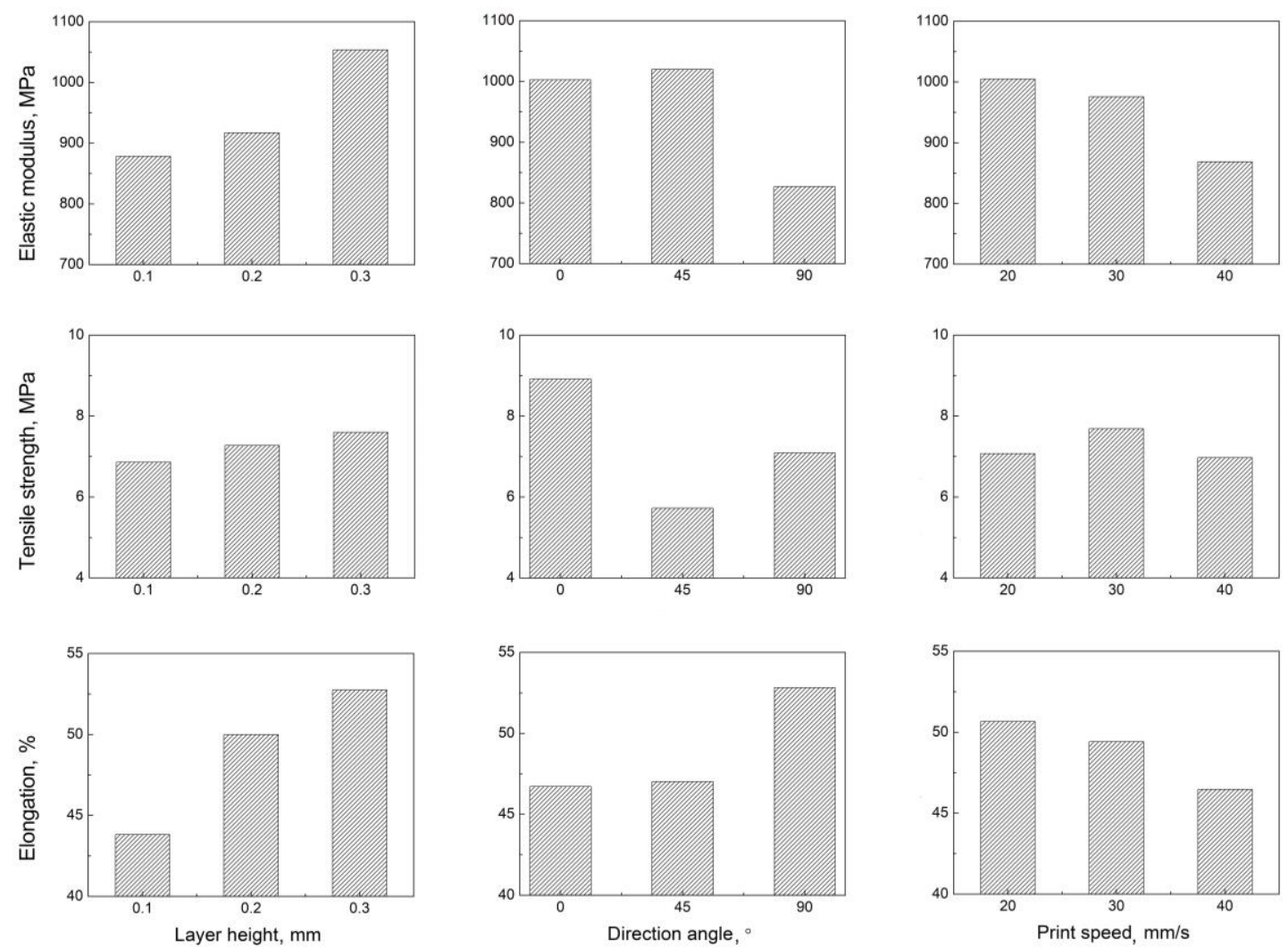

Fig. 7. Duncan multiple comparison of fixed factor levels

2. Schelly, C., Anzalone, G., Wijnen, B., Pearce, J.M. OpenSource 3-D Printing Technologies for Education: Bringing Additive Manufacturing to the Classroom Journal Visual Languages and Computing 28 2015: pp. 226-237. https://doi.org/10.1016/j.jvlc.2015.01.004

3. Stansbury, J.W., Idacavage, M.J. 3D Printing with Polymers: Challenges among Expanding Options and Opportunities Dental Materials 32 2016: pp. 54-64. https://doi.org/10.1016/j.dental.2015.09.018

4. Dudek, P. FDM 3D Printing Technology in Manufacturing Composite Elements Archieves Metallurgy and Materials 58 2013: pp. 1415-1418. https://doi.org/10.2478/amm-2013-0186

5. Ning, F., Cong, W., Qiu, J., Wei, J., Wang, S. Additive Manufacturing of Carbon Fiber Reinforced Thermoplastic Composites Using Fused Deposition Modeling Composites Part B Engineering 80 2015: pp. 369-378. https://doi.org/10.1016/j.compositesb.2015.06.013

6. Shaffer, S., Yang, K., Vargas, J., Prima, M.A.D., Voit, W. On Reducing Anisotropy in 3d Printed Polymers via Ionizing Radiation Polymer 55 2014: pp. 5969-5979. https://doi.org/10.1016/j.polymer.2014.07.054

7. Casavola, C., Cazzato, A., Moramarco, V., Pappalettere, C. Orthotropic Mechanical Properties of Fused Deposition Modelling Parts Described by Classical Laminate Theory Materials and Design 90 2016: pp. $453-458$.

https://doi.org/10.1016/j.matdes.2015.11.009

8. Tao, Y., Wang, H., Li, Z., Li, P., Shi, S. Development and Application of Wood Flour-Filled Polylactic Acid Composite Filament for 3d Printing Materials 10 2017: pp. 339-345.

https://doi.org/10.3390/ma10040339
9. Ahn, S.H., $\quad$ Montero, M., Odell, D., $\quad$ Roundy, S., Wright, P.K. Anisotropic Material Properties of Fused Deposition Modeling ABS Rapid Prototyping Journal 8 2002: pp. $248-257$. https://doi.org/10.1108/13552540210441166

10. Bellini, A., Güçeri, S. Mechanical Characterization of Parts Fabricated Using Fused Deposition Modeling Rapid Prototyping Journal 9 2003: pp. 252-264. https://doi.org/10.1108/13552540310489631

11. Sood, A.K., Ohdar, R.K., Mahapatra, S.S. Parametric Appraisal of Mechanical Property of Fused Deposition Modelling Processed Parts Materials and Design 31 2010: pp. $287-295$.

https://doi.org/10.1016/j.matdes.2009.06.016

12. Lanzotti, A., Grasso, M., Staiano, G., Martorelli, M. The Impact of Process Parameters on Mechanical Properties of Parts Fabricated in PLA with an Open-source 3-D Printer Rapid Prototyping Journal 21 2015: pp. 604-617. https://doi.org/10.1108/RPJ-09-2014-0135

13. Dawoud, M., Taha, I., Ebeid, S.J. Mechanical Behaviour of ABS: An Experimental Study Using FDM and Injection Moulding Techniques Journal Manufacturing. Processes 21 2016: pp. 39-45. https://doi.org/10.1016/j.jmapro.2015.11.002

14. ASTM D638-10. Standard Test Method for Tensile Properties of Plastics. ASTM International, West Conshohocken, PA, USA, 2010. 